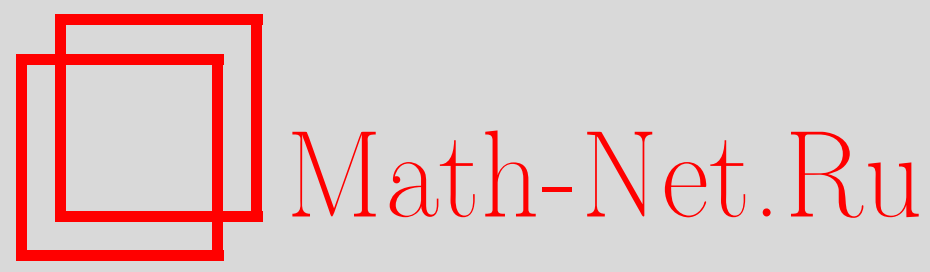

А. П. Хромов, Об обращении интегральных операторов с ядрами, разрывными на диагоналях, Матем. заметки, 1998, том 64, выпуск 6, 932-942

DOI: https://doi.org/10.4213/mzm1472

Использование Общероссийского математического портала Math-Net.Ru подразумевает, что вы прочитали и согласны с пользовательским соглашением http://www.mathnet.ru/rus/agreement

Параметры загрузки:

IP: 54.237 .206 .68

26 апреля 2023 г., 04:42:38

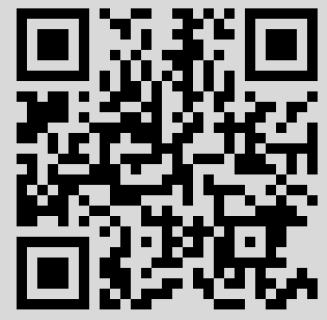




\section{ОБ ОБРАЩЕНИИ ИНТЕГРАЛЬНЫХ ОПЕРАТОРОВ \\ С ЯДРАМИ, РАЗРЫВНЫМИ НА ДИАГОНАЛЯХ}

\section{А. П. Хромов}

Найдены условия, при которых интегральный оператор

$$
A f(x)=\int_{0}^{1} A(x, t) f(t) d t
$$

с ядром $A(x, t)$, допускающим разрывы первого рода при $t=x$ и $t=1-x$, обратим. Даны точные формулы обращения, а также приложения к задаче нахождения квадратных корней из оператора $y^{\prime \prime}(x)$ с произволньми краевыми условиями и к задаче разложения по собственным функциям.

Библиографоял: 3 названия.

Рассмотрим интегральньй оператор

$$
A f(x)=\int_{0}^{1} A(x, t) f(t) d t, \quad x \in[0,1]
$$

действующий из $L[0,1]$ в $C[0,1]$. Предположим, что ядро $A(x, t)$ имеет вид

$$
\begin{aligned}
A(x, t)= & \alpha_{1} A_{1}(x, t) \varepsilon(x, t)+\alpha_{2} A_{2}(x, t) \varepsilon(t, x) \\
& +\alpha_{3} A_{3}(1-x, t) \varepsilon(1-x, t)+\alpha_{4} A_{4}(1-x, t) \varepsilon(t, 1-x),
\end{aligned}
$$

где

$$
\varepsilon(x, t)= \begin{cases}1 & \text { при } t \leqslant x, \\ 0 & \text { при } t>x,\end{cases}
$$

$A_{i}(x, t)$ непрерьвно дифференцируемы по $x$ и $A_{i}(x, t)=1+o(1), x-t \rightarrow 0$. Таким образом, ядро $A(x, t)$ непрерьвно дифференцируемо по $x$ всюду, кроме прямых $t=x$ и $t=1-x$, а на этих прямых $A(x, t)$ и $A_{x}^{\prime}(x, t)$ допускают разрывы первого рода.

Данный класс замечателен тем, что только в этом случаеможно сравнительно просто исследовать поведение резольвент Фредгольма при больших значениях спектрального параметра, что важно при спектральном анализе таких операторов. И в первую очередь (см. [1]) интересна задача точного обращения, которой посвящена настоящая статья. Для таких операторов данная задача рассматривается по-видимому впервые.

Работа выполнена при финансовой поддержке Российского фонда фундаментальных исследований, грант № 97-01-00566. 
1. Лемма 1. Если $\delta=\left(\alpha_{1}-\alpha_{2}\right)^{2}-\left(\alpha_{3}-\alpha_{4}\right)^{2} \neq 0$, mo оператор

$$
P f(x)=\left(\alpha_{1}-\alpha_{2}\right) f(x)+\left(\alpha_{4}-\alpha_{3}\right) f(1-x)
$$

обратим $u$

$$
P^{-1} g(x)=\frac{1}{\delta}\left(\left(\alpha_{1}-\alpha_{2}\right) g(x)+\left(\alpha_{3}-\alpha_{4}\right) g(1-x)\right) \text {. }
$$

ДоКАЗАТЕЛЬСТВО. Положим $P f(x)=g(x)$, т.е.

$$
\left(\alpha_{1}-\alpha_{2}\right) f(x)+\left(\alpha_{4}-\alpha_{3}\right) f(1-x)=g(x) .
$$

Меняя в (3) $x$ на $1-x$, получим

$$
\left(\alpha_{1}-\alpha_{2}\right) f(1-x)+\left(\alpha_{4}-\alpha_{3}\right) f(x)=g(1-x) .
$$

Разрешая (3), (4) как систему уравнений относительно $f(x)$ и $f(1-x)$, придем к (2).

ЛЕмма 2. Если $\delta \neq 0$ и существует $A^{-1}$, то найдется комплексное число $\beta_{0}$ такое, что оператор

$$
P^{-1}\left(\frac{d}{d x} A+\beta_{0} A\right)
$$

непрерывно обратим в $L_{2}[0,1]$.

ДоКАЗАТЕЛЬСТВо этого важного предложения аналогично доказательству теоремы 2 из [1]. Имеем

$$
\frac{d}{d x} A f(x)+\beta A f(x)=P f(x)+\widetilde{S}_{\beta} f(x),
$$

где

$$
\widetilde{S}_{\beta} f(x)=\int_{0}^{1} \widetilde{S}_{\beta}(x, t) f(t) d t, \quad \widetilde{S}_{\beta}(x, t)=A_{x}^{\prime}(x, t)+\beta A(x, t) .
$$

Отсюда по лемме 1

$$
P^{-1}\left(\frac{d}{d x} A+\beta A\right) f(x)=f(x)+S_{\beta} f(x),
$$

где $S_{\beta}=P^{-1} \widetilde{S}_{\beta}-$ интегральный оператор с ядром $S_{\beta}(x, t)$ непрерьвньм всюду, кроме прямых $t=x$ и $t=1-x$, на которых допускаются разрывы первого рода. Представим оператор $S_{\beta}$ в виде $S_{\beta}=V_{\beta}+W_{\beta}$, где $V_{\beta}$ - конечномерный оператор:

$$
V_{\beta} f(x)=\sum_{s=1}^{m_{\beta}}\left(f, \psi_{s, \beta}\right) g_{s, \beta}(x)
$$

$\left\{\psi_{s, \beta}\right\}_{1}^{m_{\beta}},\left\{g_{s, \beta}\right\}_{1}^{m_{\beta}}$ - линейно независимые системы функций,

$$
(f, g)=\int_{0}^{1} f(x) \bar{g}(x) d x,
$$

а оператор $W_{\beta}$ имеет норму $\left\|W_{\beta}\right\|<1$. Так как

$$
E+S_{\beta}=\left(E+W_{\beta}\right)\left(E+\left(E+W_{\beta}\right)^{-1} V_{\beta}\right),
$$


где $E$ - единичный оператор, вопрос об обратимости оператора $E+S_{\beta}$ сводится к вопросу об обратимости оператора $E+\left(E+W_{\beta}\right)^{-1} V_{\beta}$. Имеем

$$
\left(E+\left(E+W_{\beta}\right)^{-1} V_{\beta}\right) f(x)=f(x)+\sum_{s=1}^{m_{\beta}}\left(f, \psi_{s, \beta}\right) \widetilde{g}_{s, \beta}(x)
$$

где $\widetilde{g}_{s, \beta}(x)=\left(E+W_{\beta}\right)^{-1} g_{s, \beta}(x)$. Поэтому если

$$
f(x)+\sum_{s=1}^{m_{\beta}}\left(f, \psi_{s, \beta}\right) \widetilde{g}_{s, \beta}(x)=g(x)
$$

Tо

$$
f(x)=g(x)+\sum_{s=1}^{m_{\beta}} \gamma_{s} \widetilde{g}_{s, \beta}(x) .
$$

Подставляя последнее равенство в (5), получим

$$
g(x)+\sum_{s=1}^{m_{\beta}} \gamma_{s} \widetilde{g}_{s, \beta}(x)+\sum_{s=1}^{m_{\beta}}\left(g+\sum_{j=1}^{m_{\beta}} \gamma_{j} \widetilde{g}_{j, \beta}, \psi_{s, \beta}\right) \widetilde{g}_{s, \beta}(x)=g(x)
$$

Отсюда получаем систему

$$
\gamma_{s}+\sum_{j=1}^{m_{\beta}} \gamma_{j}\left(\widetilde{g}_{j, \beta}, \psi_{s, \beta}\right)=-\left(g, \psi_{s, \beta}\right), \quad s=1, \ldots, m_{\beta}
$$

Система (6) эквивалентна (5). Необходимым и достаточньм условием однозначной разрешимости системы (6) является

$$
\Delta(\beta)=\operatorname{det}\left\|\delta_{s, j}+\left(\widetilde{g}_{j, \beta}, \psi_{s, \beta}\right)\right\|_{1}^{m_{\beta}} \neq 0
$$

где $\delta_{s, j}-$ символ Кронекера. Предположим, что $E+S_{\beta}$ необратим ни при каком $\beta$. Тогда $\Delta(\beta) \equiv 0$ и при любом $\beta$ существует функция $f_{\beta}(x) \in L_{2}[0,1], f_{\beta}(x) \neq 0$ почти всюду, такая, что $f_{\beta}+S_{\beta} f_{\beta}=0$. Отсюда следует, что

$$
\left(\frac{d}{d x} A+\beta A\right) f(x)=0 .
$$

Значит, $A f_{\beta}=C(\beta) e^{-\beta x}$. Покажем, что хотя бы при одном $\beta C(\beta)=0$. Допустим противное: пусть $C(\beta) \neq 0$ при всех $\beta$. Тогда

$$
P^{-1} \frac{d}{d x} A f_{\beta}=f_{\beta}+S_{0} f_{\beta}=C(\beta)(-\beta) P^{-1}\left(e^{-\beta x}\right) .
$$

Но $f_{\beta}+S_{0} f_{\beta}=f_{\beta}+V_{0} f_{\beta}+W_{0} f_{\beta}$. Поэтому

$$
f_{\beta}+\left(E+W_{0}\right)^{-1} V_{0} f_{\beta}=C(\beta)(-\beta) g_{1},
$$


где $g_{1}(x)=\left(E+W_{0}\right)^{-1} P^{-1}\left(e^{-\beta x}\right)$. Действительно,

$$
f_{\beta}(x)+\sum_{s=1}^{m_{0}}\left(f_{\beta}, \psi_{s, 0}\right) \widetilde{g}_{s, 0}(x)=C(\beta)(-\beta) g_{1}(x) .
$$

Отсюда

$$
\left(f_{\beta}, \psi_{j, 0}\right)+\sum_{s=1}^{m_{0}}\left(f_{\beta}, \psi_{s, 0}\right)\left(\widetilde{g}_{s, 0}, \psi_{j, 0}\right)=C(\beta)(-\beta)\left(g_{1}, \psi_{j, 0}\right), \quad j=1, \ldots, m_{0} .
$$

Определитель этой системы $\Delta(0)=0$. Значит, существуют компоненты $\xi_{k}, \sum_{1}^{m_{0}}\left|\xi_{k}\right|>0$, не зависяшие от $\beta$, такие, что $C(\beta)(-\beta)\left(g_{1}, \psi\right)=0$, где $\psi(x)=\sum_{j} \xi_{j} \psi_{j, 0}(x)$. Отсюда $\left(g_{1}, \psi\right) \equiv 0$ по $\beta$. Значит, $\left(e^{-\beta x}, \widetilde{\psi}\right) \equiv 0$, где $\widetilde{\psi}(x)=\left(P^{-1}\right)^{k}\left(E+W_{0}^{*}\right)^{-1} \psi$. Отсюда $\widetilde{\psi}(x)=0$ почти всюду. Значит, и $\psi(x)=0$ почти всюду. Противоречие. Поэтому существует $\beta_{0}$ такое, что $A f_{\beta_{0}}=0$. Отсюда $f_{\beta_{0}}=0$ почти всюду. Противоречие. Лемма доказана.

Теорема 1. Пусть $\delta \neq 0$ и существует $A^{-1}$. Если $y(x)=A f(x)$, где $f(x) \in L[0,1]$, mo

$$
\begin{gathered}
f(x)=\left(E+S_{\beta_{0}}\right)^{-1} P^{-1}\left(y^{\prime}(x)+\beta_{0} y(x)\right) \\
y(0)=\int_{0}^{1} A(0, t)\left(E+S_{\beta_{0}}\right)^{-1} P^{-1}\left(y^{\prime}(t)+\beta_{0} y(t)\right) d t
\end{gathered}
$$

и обратно, из (7), (8) следует, что $y(x)=A f(x)$.

ДоКАЗАТЕЛЬСТво. Пусть сначала $f(x) \in L_{2}[0,1]$. Тогда

$$
\left(E+S_{\beta_{0}}\right) f(x)=P^{-1}\left(\frac{d}{d x} A+\beta_{0} A\right) f(x)=P^{-1}\left(y^{\prime}(x)+\beta_{0} y(x)\right) .
$$

Отсюда по лемме 2 получаем (7). Далее, в силу (7) из

$$
y(0)=\int_{0}^{1} A(0, t) f(t) d t
$$

получаем (8).

Обратно, пусть вьполнены (7) и (8). Тогда

$$
y(0)=\int_{0}^{1} A(0, t) f(t) d t .
$$

Далее,

$$
P\left(E+S_{\beta_{0}}\right) f(x)=y^{\prime}(x)+\beta_{0} y(x)
$$

$\mathrm{Ho}$

$$
P\left(E+S_{\beta_{0}}\right) f(x)=\frac{d}{d x} A f(x)+\beta_{0} A f(x) .
$$

Значит, если положим $z(x)=y(x)-A f(x)$, то из (9)-(11) получим $z^{\prime}(x)+\beta_{0} z(x)=0$, $z(0)=0$. Отсюда $z(x) \equiv 0$, т.е. $y(x)=A f(x)$.

Чтобы убедиться в справедливости соотношений $(7),(8)$ для $f(x) \in L[0,1]$, необходимо записать их для последовательности $\left\{f_{n}(x)\right\}$, где $f_{n}(x) \in L_{2}[0,1]$ и $\left\|f_{n}-f\right\|_{L[0,1]} \rightarrow 0$, и затем перейти к пределу. Теорема доказана. 
2. Условие существования $A^{-1}$ трудно проверяемо. Поэтому укажем частные случаи, когда существование $A^{-1}$ очевидно.

Прежде всего отметим следующий случай: все $\alpha_{i}$, кроме одного, равны 0 . Пусть, например, $\alpha_{1}=\alpha_{2}=\alpha_{4}=0, \alpha_{3} \neq 0$. В этом случае формула обращения легко получается и имеет вид

$$
A^{-1} y=-\frac{1}{\alpha_{3}}(E+N)^{-1} y^{\prime}(1-x), \quad y(1)=0,
$$

где

$$
N f(x)=\int_{0}^{x} N(x, t) f(t) d t, \quad N(x, t)=\frac{\partial}{\partial x} A_{3}(x, t) .
$$

Кроме того, $A^{-1}$ существует, если все $\alpha_{i}$, кроме одного, достаточно малы. Мы не приводим здесь формулу для $A^{-1}$ ввиду ее громоздкости.

Рассмотрим теперь менее тривиальный случай.

Tеорема 2. Пусть $\alpha_{2}=\alpha_{4}=0, \delta \neq 0 u A_{1}(x, t) \equiv A_{3}(x, t)$. Тогда $A^{-1}$ cyществует, область определения $D_{A^{-1}}$ оператора $A^{-1}$ состоит из абсолютно непрерывных функиий, удовлетворяющих граничному условию

$$
\alpha_{1} y(0)-\alpha_{3} y(1)=0
$$

и справедлива формула

$$
A^{-1} y=\frac{1}{\delta}(E+N)^{-1}\left(\alpha_{1} y^{\prime}(x)+\alpha_{3} y^{\prime}(1-x)\right)
$$

$2 \partial e$

$$
N f(x)=\int_{0}^{x} N(x, t) f(t) d t, \quad N(x, t)=\frac{\partial}{\partial x} A_{1}(x, t) .
$$

ДоКАЗАТЕЛЬСТВо. Положим $y(x)=A f(x)$. Тогда

$$
y(0)=\alpha_{3} \int_{0}^{1} A_{3}(1, t) f(t) d t, \quad y(1)=\alpha_{1} \int_{0}^{1} A_{1}(1, t) f(t) d t
$$

Отсюда следует (12). Далее, дифференцируя $y(x)=A f(x)$, получим

$$
y^{\prime}(x)=\alpha_{1} f(x)-\alpha_{3} f(1-x)+\alpha_{1} \int_{0}^{x} N(x, t) f(t) d t-\alpha_{3} \int_{0}^{1-x} N(1-x, t) f(t) d t .
$$

Меняя $x$ на $1-x$, получим

$$
y^{\prime}(1-x)=\alpha_{1} f(1-x)-\alpha_{3} f(x)+\alpha_{1} \int_{0}^{1-x} N(1-x, t) f(t) d t-\alpha_{3} \int_{0}^{x} N(x, t) f(t) d t
$$

Умножая (14) на $\alpha_{1},(15)$ на $\alpha_{3}$ и складьвая, получим

$$
\alpha_{1} y^{\prime}(x)+\alpha_{3} y^{\prime}(1-x)=\delta(f(x)+N f(x)) .
$$

Отсюда следует (13). 
Обратно, обозначим через $f(x)$ правую часть (13). Тогда получаем (16). Меняя в (16) $x$ на $1-x$, находим

$$
\alpha_{1} y^{\prime}(1-x)+\alpha_{3} y^{\prime}(x)=\delta\left(f(1-x)+\int_{0}^{1-x} N(1-x, t) f(t) d t\right) .
$$

Из (16), умноженного на $\alpha_{1}$, вычтем (17), умноженное на $\alpha_{3}$, и поделим полученньй результат на $\delta$. В итоге

$$
y^{\prime}(x)=\frac{d}{d x} A f(x) .
$$

Отсюда, интегрируя от 0 до $x$ и от $x$ до 1 , получаем

$$
y(x)-y(0)=A f(x)-A f(0), \quad y(1)-y(x)=A f(1)-A f(x) .
$$

Умножая первое соотношение в (18) на $\alpha_{1}$, второе на $\alpha_{3}$ и складьвая, в силу (12) и равенства $\alpha_{1} A f(0)=\alpha_{3} A f(1)$ получаем $\left(\alpha_{1}-\alpha_{3}\right) y(x)=\left(\alpha_{1}-\alpha_{3}\right) A f(x)$. Теорема доказана.

Аналогично доказывается

Teорема 3. Пусть $\alpha_{1}=\alpha_{3}=0, \delta \neq 0 u A_{2}(x, t) \equiv A_{4}(x, t)$. Tогдa $A^{-1} c y$ ществует, $D_{A^{-1}}$ состоит из абсолютно непрерывных функций, удовлетворяющ, граничному условию

$$
\alpha_{4} y(0)-\alpha_{2} y(1)=0
$$

и справедлива формула

$2 \partial e$

$$
A^{-1} y=-\frac{1}{\delta}(E-N)^{-1}\left(\alpha_{2} y^{\prime}(x)+\alpha_{4} y^{\prime}(1-x)\right),
$$

$$
N f(x)=\int_{x}^{1} N(x, t) f(t) d t, \quad N(x, t)=\frac{\partial}{\partial x} A_{2}(x, t) .
$$

Пусть теперь $\alpha_{2}=\alpha_{4}=0, \delta \neq 0$, но $A_{1}(x, t) \not \equiv A_{3}(x, t)$. В этом случае о существовании $A^{-1}$ ничего не известно. Кроме того, если интегрирование идет не от 0, а от $1 / 2$, то получаем интересньй класс вольтерровых операторов. Итак, пусть

$$
A f(x)=\alpha_{1} \int_{1 / 2}^{x} A_{1}(x, t) f(t) d t+\alpha_{3} \int_{1 / 2}^{1-x} A_{3}(1-x, t) f(t) d t .
$$

Теорема 4. Если $\delta \neq 0$, то $A^{-1}$ существует, $D_{A^{-1}}$ состоит из абсолютно непрерывных функций, удовлетворяющих граничному условию $y(1 / 2)=0, u$ справедлива формула $A^{-1} y(x)=\beta_{11} y^{\prime}(x)-\beta_{12} y^{\prime}(1-x)+\int_{1 / 2}^{x} N_{1,1}(x, t) y^{\prime}(t) d t+\int_{1 / 2}^{1-x} N_{1,2}(x, 1-t) y^{\prime}(t) d t$. Здесь $\left\{\beta_{11}, \beta_{12}\right\}$ - первая строка матрииь $B^{-1}$, обратной $\kappa$

$$
B=\left(\begin{array}{ll}
\alpha_{1} & -\alpha_{3} \\
\alpha_{3} & -\alpha_{1}
\end{array}\right)
$$

$N_{i, j}(x, t)$ - компоненты ядра оператора

$$
N=\left(B+\int_{1 / 2}^{x} \widetilde{A}_{x}^{\prime}(x, t) d t\right)^{-1}-B^{-1}
$$

в пространстве $L_{2}[0,1] \times L_{2}[0,1] u$

$$
\widetilde{A}(x, t)=\left(\begin{array}{ll}
\alpha_{1} A_{1}(x, t) & -\alpha_{3} A_{3}(1-x, t) \\
\alpha_{3} A_{3}(x, t) & -\alpha_{1} A_{1}(1-x, t)
\end{array}\right) .
$$


ДОКАЗАТЕЛЬСТВО. Положим $y(x)=A f(x)$. Тогда

$$
\begin{gathered}
y(x)=\alpha_{1} \int_{1 / 2}^{x} A_{1}(x, t) f(t) d t-\alpha_{3} \int_{1 / 2}^{x} A_{3}(1-x, 1-t) f(1-t) d t, \\
y(1-x)=\alpha_{3} \int_{1 / 2}^{x} A_{3}(x, t) f(t) d t-\alpha_{1} \int_{1 / 2}^{x} A_{1}(1-x, 1-t) f(1-t) d t .
\end{gathered}
$$

Положим $y_{1}(x)=y(x), y_{2}(x)=y(1-x), f_{1}(x)=f(x), f_{2}(x)=f(1-x), Y(x)=\left\{y_{1}(x)\right.$, $\left.y_{2}(x)\right\}^{T}, F(x)=\left\{f_{1}(x), f_{2}(x)\right\}^{T}$. Тогда равенства (19) запишутся следующим образом:

$$
Y(x)=\int_{1 / 2}^{x} \widetilde{A}(x, t) F(t) d t .
$$

Рассмотрим (20). Прежде всего покажем, что если $f_{2}(x)=f_{1}(1-x)$, то и $y_{2}(x)=$ $y_{1}(1-x)$. В самом деле, в этом случае

$$
\begin{aligned}
y_{2}(1-x) & =\alpha_{3} \int_{1 / 2}^{1-x} A_{3}(1-x, t) f_{1}(t) d t-\alpha_{1} \int_{1 / 2}^{1-x} A_{1}(x, 1-t) f_{1}(1-t) d t \\
& =-\alpha_{3} \int_{1 / 2}^{x} A_{3}(1-x, 1-t) f_{1}(1-t) d t+\alpha_{1} \int_{1 / 2}^{x} A_{1}(x, t) f(t) d t=y_{1}(x) .
\end{aligned}
$$

Теперь снова посмотрим на (20) как на матричную запись (19). Тогда $Y(1 / 2)=0$ и, дифференцируя (20), получаем

$$
\left(B^{-1}+N\right) Y^{\prime}(x)=F(x) .
$$

Первая строка (21) дает

$$
\beta_{11} y^{\prime}(x)-\beta_{12} y^{\prime}(1-x)+\int_{1 / 2}^{x} N_{1,1}(x, t) y^{\prime}(t) d t+\int_{1 / 2}^{1-x} N_{1,2}(x, 1-t) y^{\prime}(t) d t=f(x) .
$$

Обратно, пусть имеет место (22) и $y(1 / 2)=0$. Для $F(x)=\left\{f_{1}(x), f_{2}(x)\right\}^{T}$, где $f_{2}(x)=$ $f_{1}(1-x)=f(1-x)$, положим

$$
Z(x)=\int_{1 / 2}^{x} \widetilde{A}(x, t) F(t) d t .
$$

Тогда обязательно $z_{2}(1-x)=z_{1}(x)$ и по-доказанному $z(x)=z_{1}(x)$ удовлетворяет уравнению (22) и $z(1 / 2)=0$. Положим $v(x)=z(x)-y(x)$. Тогда $v(x)$ удовлетворяет (22) при $f(x) \equiv 0$. Покажем, что $v(x) \equiv 0$. Положим $w_{1}(x)=v^{\prime}(x), w_{2}(x)=v^{\prime}(1-x)$. Тогда

$$
\beta_{11} w_{1}(x)-\beta_{12} w_{2}(x)+\int_{1 / 2}^{x} N_{1,1}(x, t) w_{1}(t) d t-\int_{1 / 2}^{x} N_{1,2}(x, t) w_{2}(t) d t=0 .
$$

Меняя в (23) $x$ на $1-x$, придем к соотношению

$$
\beta_{12} w_{1}(x)-\beta_{11} w_{2}(x)-\int_{1 / 2}^{x} N_{1,2}(1-x, 1-t) w_{1}(t) d t+\int_{1 / 2}^{x} N_{1,1}(1-x, 1-t) w_{2}(t) d t=0 .
$$

Так как $\beta_{11}^{2}-\beta_{12}^{2}=\delta^{-1} \neq 0$, из (23) и (24) следует, что $w(x) \equiv 0$. А так как $v(1 / 2)=0$, то и $v(x) \equiv 0$. Значит, $y(x)=A f(x)$. Теорема доказана. 
ЗАмЕчАниЕ. Аналогичньй результат имеет место и для оператора

$$
A f(x)=\alpha_{2} \int_{x}^{1 / 2} A_{2}(x, t) f(t) d t+\alpha_{4} \int_{1-x}^{1 / 2} A_{4}(1-x, t) f(t) d t, \quad \alpha_{2}^{2} \neq \alpha_{4}^{2} .
$$

Наконец, отметим еще один результат, которьй доказьвается аналогично теореме 2.

Tеорема 5. Пусть $A_{i}(x, t) \equiv 1(i=1,2,3,4) u \delta \neq 0$. Тогда $A^{-1}$ существует, $u$ если $y(x)=A f(x)$, mo

$$
\begin{gathered}
A^{-1} y(x)=\frac{1}{\delta}\left(\left(\alpha_{1}-\alpha_{2}\right) y^{\prime}(x)+\left(\alpha_{3}-\alpha_{4}\right) y^{\prime}(1-x)\right)=f(x), \\
U(y)=\left(\alpha_{1}+\alpha_{4}\right) y(0)-\left(\alpha_{2}+\alpha_{3}\right) y(1)=0,
\end{gathered}
$$

и обратно.

3. Укажем на одно применение дифференциально-разностного оператора, входящего в $A^{-1} y$. Рассмотрим в $L_{2}[0,1]$ следующий дифференциально-разностный оператор $\mathscr{L}:$

$$
l[y]=\beta_{1} y^{\prime}(x)+\beta_{2} y^{\prime}(1-x), \quad U(y)=c_{0} y(0)-d_{0} y(1)=0 .
$$

Выражение $l[y]$ замечательно тем, что $l^{2}[y]=\delta y^{\prime \prime}(x)$, где $\delta=\beta_{1}^{2}-\beta_{2}^{2}$. Это обстоятельство позволяет исследовать квадратные корни из оператора дифференцирования второго порядка с произвольными граничньми условиями

$$
U_{i}(y)=c_{i} y^{(i)}(0)-d_{i} y^{(i)}(1)=0, \quad i=0,1 .
$$

Обозначим через $D$ множество функций $y(x)$, абсолютно непрерьвных вместе с первой производной, $y^{\prime \prime}(x) \in L_{2}[0,1]$, удовлетворяющих граничным условиям (26).

Теорема 6. Для того чтобы $D=D_{\mathscr{L}^{2}}$ при некоторых $\beta_{1} u \beta_{2}$, причем $\delta=\beta_{1}^{2}-\beta_{2}^{2}$ $\neq 0$, необходимо и достаточно, чтобы выполнялось одно из трех условий:

a) $c_{0}^{2} \neq d_{0}^{2}, c_{1}^{2} \neq d_{1}^{2}$

б) $c_{0}=d_{0}, c_{1}=d_{1}$;

в) $c_{0}=-d_{0}, c_{1}=-d_{1}$.

ДоКАЗАТЕЛЬСТво. Необходимость. Если $y(x) \in D_{\mathscr{L}^{2}}$, то

$$
U(y)=c_{0} y(0)-d_{0} y(1)=0, \quad U(l[y])=\left(c_{0} \beta_{1}-d_{0} \beta_{2}\right) y^{\prime}(0)-\left(d_{0} \beta_{1}-c_{0} \beta_{2}\right) y^{\prime}(1)=0 .
$$

Сравнивая эти условия с (26), имеем

$$
c_{0} \beta_{1}-d_{0} \beta_{2}=c_{1}, \quad d_{0} \beta_{1}-c_{0} \beta_{2}=d_{1}
$$

Из этих соотношений легко получаем

$$
\left(c_{0}^{2}-d_{0}^{2}\right) \delta=c_{1}^{2}-d_{1}^{2} .
$$

Отсюда видно, что если $c_{0}^{2} \neq d_{0}^{2}$, то и $c_{1}^{2} \neq d_{1}^{2}$, и обратно. 
Пусть теперь $c_{0}^{2}=d_{0}^{2}$ и $c_{1}^{2}=d_{1}^{2}$ одновременно. В этом случае, умножая первое соотношение из $(27)$ на $d_{0}$, второе на $c_{0}$ и вычитая из первого второе, получим $c_{1} d_{0}-c_{0} d_{1}=0$. Отсюда следует б) или в).

Достаточность. Пусть $c_{0}, c_{1}, d_{0}, d_{1}$ таковы, что вьполняется одно из условий а), б) или в). Покажем, что найдутся $\beta_{1}$ и $\beta_{2}$ такие, что $\delta=\beta_{1}^{2}-\beta_{2}^{2} \neq 0$ и вьполняются (27). Пусть сначала вьполняется а). Рассмотрим систему (27). Она однозначно разрешима относительно $\beta_{1}$ и $\beta_{2}$, и из (28) следует, что $\delta \neq 0$. Пусть теперь вьполняется б). Тогда система (27) преврашается в одно уравнение $c_{0} \beta_{1}-d_{0} \beta_{2}=c_{1}$, которое всегда разрешимо, причем неоднозначно. Поэтому всегда можно выбрать это решение так, чтобы $\delta \neq 0$. Аналогично рассматривается случай в). Теорема доказана.

Рассмотрим частньй случай оператора $\mathscr{L}$, когда $\mathscr{L}=A^{-1}$ из теоремы 5.

ТЕорема 7. Для того чтобъ $D=D_{A^{-2}}$, где $A^{-1}$ определяется из (25) nри некоторых $\alpha_{i}(i=1,2,3,4)$, причем $\delta=\left(\alpha_{1}-\alpha_{2}\right)^{2}-\left(\alpha_{3}-\alpha_{4}\right)^{2} \neq 0$, необходимо $u$ достаточно, чтобы $c_{1}+d_{1}=c_{0}^{2}-d_{0}^{2}$ и выполнялось одно из двух условий:

a) $c_{0}^{2} \neq d_{0}^{2}, c_{1}^{2} \neq d_{1}^{2}$

б) $c_{0}=-d_{0}, c_{1}=-d_{1}$.

ДокАЗАТЕЛЬСТво. Необходимость. Если $y(x)=A^{2} f(x)$ и $\delta \neq 0$, то

$$
\begin{gathered}
\frac{1}{\delta} y^{\prime \prime}(x)=f(x), \\
\left(\alpha_{1}+\alpha_{4}\right) y(0)-\left(\alpha_{2}+\alpha_{3}\right) y(1)=0, \\
\left(\left(\alpha_{1}+\alpha_{4}\right)\left(\alpha_{1}-\alpha_{2}\right)-\left(\alpha_{2}+\alpha_{3}\right)\left(\alpha_{3}-\alpha_{4}\right)\right) y(0) \\
+\left(\left(\alpha_{1}+\alpha_{4}\right)\left(\alpha_{3}-\alpha_{4}\right)-\left(\alpha_{2}+\alpha_{3}\right)\left(\alpha_{1}-\alpha_{2}\right)\right) y(1)=0 .
\end{gathered}
$$

Совпадение условий (26) и (29) ведет к разрешимости относительно $\alpha_{i}$ системы

$$
\begin{gathered}
\alpha_{1}+\alpha_{4}=c_{0}, \quad \alpha_{2}+\alpha_{3}=d_{0}, \\
\left(\alpha_{1}+\alpha_{4}\right)\left(\alpha_{1}-\alpha_{2}\right)-\left(\alpha_{3}+\alpha_{4}\right)\left(\alpha_{3}-\alpha_{4}\right)=c_{1} \\
\left(\alpha_{1}+\alpha_{4}\right)\left(\alpha_{3}-\alpha_{4}\right)-\left(\alpha_{2}+\alpha_{3}\right)\left(\alpha_{1}-\alpha_{2}\right)=-d_{1} .
\end{gathered}
$$

Система (30) эквивалентна следующей линейной системе:

$$
\begin{gathered}
\alpha_{1}+\alpha_{4}=c_{0}, \quad \alpha_{2}+\alpha_{3}=d_{0}, \\
c_{0}\left(\alpha_{1}-\alpha_{2}\right)-d_{0}\left(\alpha_{3}-\alpha_{4}\right)=c_{1}, \quad c_{0}\left(\alpha_{3}-\alpha_{4}\right)-d_{0}\left(\alpha_{1}-\alpha_{2}\right)=-d_{1} .
\end{gathered}
$$

Рассмотрим систему (31). Вычитая из третьего уравнения четвертое, получим

$$
\left(c_{0}+d_{0}\right)\left(\alpha_{1}-\alpha_{2}+\alpha_{4}-\alpha_{3}\right)=c_{1}+d_{1}
$$

а вычитая из первого уравнения второе -

$$
\alpha_{1}-\alpha_{2}+\alpha_{4}-\alpha_{3}=c_{0}-d_{0}
$$

Из (32) и (33) находим

$$
c_{0}^{2}-d_{0}^{2}=c_{1}+d_{1}
$$


Теперь в силу теоремы 6 должно вьполняться одно из трех ее условий а), б) или в). Ясно, что условие б) и (34) противоречивы. Поэтому может вьполняться лишь условие а) или в) теоремы 6.

Достаточность. Пусть теперь выполняется (34) и одно из двух условий а) или б). Положим

$$
\alpha_{1}+\alpha_{4}=c_{0}, \quad \alpha_{2}+\alpha_{3}=d_{0} .
$$

Тогда в силу (34) имеем (32). Потребуем дополнительно

$$
c_{0}\left(\alpha_{1}-\alpha_{2}\right)-d_{0}\left(\alpha_{3}-\alpha_{4}\right)=c_{1}
$$

Из условия а) или б) следует, что ранг системы (35), (36) равен 3 , поэтому она совместна. Из (32) и (34) получаем $d_{0}\left(\alpha_{1}-\alpha_{2}\right)+c_{0}\left(\alpha_{4}-\alpha_{3}\right)=d_{1}$. Тем самьм, получаем $(31)$. Покажем, что $\delta \neq 0$. Имеем $\left(c_{0}^{2}-d_{0}^{2}\right) \delta=c_{1}^{2}-d_{1}^{2}$. Поэтому в случае а) $\delta \neq 0$. Пусть теперь выполняется б). В этом случае (31) переходит в

$$
\alpha_{1}+\alpha_{4}=c_{0}, \quad \alpha_{2}+\alpha_{3}=-c_{0}, \quad c_{0}\left(\alpha_{1}-\alpha_{2}\right)+c_{0}\left(\alpha_{3}-\alpha_{4}\right)=c_{1} .
$$

Отсюда получаем

$$
\alpha_{1}-\alpha_{2}+\alpha_{4}-\alpha_{3}=2 c_{0}, \quad \alpha_{1}-\alpha_{2}+\alpha_{3}-\alpha_{4}=\frac{c_{1}}{c_{0}}
$$

Значит, и в этом случае $\delta=2 c_{1} \neq 0$. Теорема доказана.

СлЕДСТВИЕ. Если $c_{1}+d_{1}=c_{0}^{2}-d_{0}^{2}$ и выполняется одно из условий а) или б) теоремы 7, то функиия Грина $G(x, t)$ оператора $y^{\prime \prime}(x)$ с краевыми условиями (26) существует и существуют $\alpha_{i}(i=1,2,3,4)$ такие, что

$$
\delta=\left(\alpha_{1}-\alpha_{2}\right)^{2}-\left(\alpha_{3}-\alpha_{4}\right)^{2} \neq 0, \quad G(x, t)=\frac{1}{\delta} \int_{0}^{1} A(x, \tau) A(\tau, t) d \tau,
$$

где $A(x, t)$ - ядро оператора $(1)$, когда $A_{i}(x, t) \equiv 0(i=1,2,3,4)$.

Рассмотрим более общую ситуацию. Пусть

$$
l[y]=\beta_{1} y^{\prime}(x)+\beta_{2} y^{\prime}(1-x)+\beta_{3} y(1-x) .
$$

Тогда $l^{2}[y]=\delta y^{\prime \prime}(x)+\beta_{3}^{2} y(x)$, где $\delta=\beta_{1}^{2}-\beta_{2}^{2}$. Обозначим через $D$ множество функций, абсолютно непрерывных вместе с первой производной, $y^{\prime \prime}(x) \in L_{2}[0,1]$, удовлетворяющих краевьг условиям

$$
U_{0}(y)=c_{0} y(0)-d_{0} y(1)=0, \quad U_{1}(y)=c_{1} y^{\prime}(0)-d_{1} y^{\prime}(1)+c_{2} y(0)-d_{2} y(1)=0 .
$$

Обозначим через $\mathscr{L}$ оператор $(37)$ с краевым условием $U_{0}(y)=0$.

Аналогично теореме 6 доказьвается

Теорема 8. Для того чтобы $D=D_{\mathscr{L}^{2}}$ при некоторых $\beta_{1}, \beta_{2}, \beta_{3}$, причем $\delta \neq 0$, необходимо и достаточно, чтобы $\left|c_{1}\right|+\left|d_{1}\right|>0$ и выполнялось одно из трех условий а), б) или в) теоремы 6. 
4. В качестве другого приложения результатов п. 1, 2 приведем следующий результат, полученньй совместно с А. П. Гуревичем в [2].

Теорема 9. Если ядро $A(x, t)$ оператора

$$
A f(x)=\int_{0}^{1-x} A(1-x, t) f(t) d t
$$

трижды непрерывно дифферениируемо по $x$ и дважды по $t$,

$$
A(x, x) \equiv 1,\left.\quad \frac{\partial}{\partial x} A(x, t)\right|_{t=x} \equiv 0,
$$

то для любой функиии $f(x) \in L[0,1]$ справедливо соотношение

$$
\lim _{r \rightarrow \infty} \max _{0<\delta \leqslant x \leqslant 1-\delta}\left|S_{r}(f, x)-\sigma_{r}(f, x)\right|=0,
$$

где $S_{r}(f, x)$ - частная сумма ряда Фурье по собственным и присоединенным функииям оператора $A$ для $k$ таких, что $\left|\lambda_{k}\right|<r^{2}\left(\lambda_{k}-\right.$ характеристическое число оператора $A, \sigma_{r}(f, x)$ - частичная сумма тригонометрического ряда Фурье для $k$ таких, что $|k \pi|<r)$.

Доказательство сводится к простой проверке условий теоремы 5 из [1] для ядра оператора $A^{2}$.

Без доказательств результаты статьи частично опубликованы в [2] и [3].

\section{СПИСОК ЦИТИРОВАННОЙ ЛИТЕРАТУРЫ}

[1] Хромов А. П. Теоремы равносходимости для интегральных операторов // Матем. сб. 1981. T. 114 (156). №6 6. C. 378-405.

[2] Хромов А. П., Гуревич А. П. Теоремы равносходимости для одного класса интегральных операторов // Восьмая Саратовская зимняя школа. Тезисы докл. Саратов, 1996. С. 117.

[3] Хромов А.П. Об обращении одного класса интегральных операторов // Восьмая Саратовская зимняя школа. Тезисы докл. Саратов, 1996. С. 118.

Саратовский государственньй университет

Поступило

E-mail: khromov@scnit.saratov.su

05.01 .97

Исправленный вариант 08.09.97 